\title{
Inclusion of a pH-responsive amino acid-based amphiphile in methotrexate- loaded chitosan nanoparticles as a delivery strategy in cancer therapy
}

Daniele Rubert Nogueira ${ }^{1,2, *}$, Laís E. Scheeren ${ }^{1,2}$, Letícia B. Macedo ${ }^{1}$, Ana Isa P. Marcolino ${ }^{1,2}$, M. Pilar Vinardell ${ }^{3}$, Montserrat Mitjans ${ }^{3}$, M. Rosa Infante ${ }^{4}$, Ammad A. Farooqi ${ }^{5}$, Clarice M. B. Rolim ${ }^{1,2}$

${ }^{1}$ Departamento de Farmácia Industrial, Universidade Federal de Santa Maria, Av. Roraima 1000, 97105-900, Santa Maria, RS, Brazil

${ }^{2}$ Programa de Pós-Graduação em Ciências Farmacêuticas, Universidade Federal de Santa Maria, Av. Roraima 1000, 97105-900, Santa Maria, RS, Brazil

${ }^{3}$ Departament de Fisiologia, Facultat de Farmàcia, Universitat de Barcelona, Av. Joan XXIII s/n, 08028, Barcelona, Spain

${ }^{4}$ Departamento de Tecnología Química y de Tensioactivos, IQAC, CSIC, C/ Jordi Girona 1826, 08034, Barcelona, Spain

${ }^{5}$ Laboratory for Translational Oncology and Personalized Medicine, Rashid Latif Medical College, 35 Km Ferozepur Road, Lahore, Pakistan

* Corresponding author: Phone: +55 553220 9548; Fax: +55 5532208248

E-mail address: daniele.rubert@gmail.com (Daniele Rubert Nogueira). 


\begin{abstract}
The encapsulation of antitumor drugs in nanosized systems with $\mathrm{pH}$-sensitive behavior is a promissing approach that may enhance the success of chemotherapy in many cancers. The nanocarrier dependence on $\mathrm{pH}$ might trigger an efficient delivery of the encapsulated drug both in the acidic extracellular environment of tumors and, especially, in the intracellular compartments through disruption of endosomal membrane. In this context, here we reported the preparation of chitosan-based nanoparticles encapsulating methotrexate as a model drug (MTXCS-NPs), which comprises the incorporation of an amino acid-based amphiphile with $\mathrm{pH}$ responsive properties $(77 \mathrm{KS})$ on the ionotropic complexation process. The presence of $77 \mathrm{KS}$ clearly gives a pH-sensitive behavior to NPs, which allowed accelerated release of MTX with decreasing $\mathrm{pH}$ as well as $\mathrm{pH}$-dependent membrane-lytic activity. This latter performance demonstrates the potential of these NPs to facilitate cytosolic delivery of endocytosed materials. Outstandingly, the cytotoxicity of MTX-loaded CS-NPs was higher than free drug to MCF-7 tumor cells and, to a lesser extent, to HeLa cells. Based on the overall results, MTX-CS-NPs modified with the $\mathrm{pH}$-senstive surfactant $77 \mathrm{KS}$ could be potentially useful as a carrier system for intracellular drug delivery and, thus, a promising targeting anticancer chemotherapeutic agent.
\end{abstract}

Keywords: Chitosan nanoparticles; In vitro antitumor activity; Lysine-based surfactant Methotrexate; $\mathrm{pH}$-sensitivity 


\section{Introduction}

Cancer is one of the main causes of mortality worldwide and the World Health Organization (WHO) estimates that there will be 15 million new cases of cancer worldwide in 2020 (ETPN 2015). The clinical efficacy of the conventional treatments is often compromised by the acquisition of resistance in cancer cells and/or by the generation of several side effects to the patients (Banerjee et al. 2002; Dong and Mumper 2010). In this context, the nanotechnology-based pharmaceutical products might provide a wide range of new tools and possibilities in cancer therapy, from earlier diagnostics to more efficient and more targeted treatments (Chen et al. 2014; Das and Sahoo 2011; Li et al. 2012).

Methotrexate (MTX) is a cytotoxic drug used in the therapy of solid tumors and leukemias. Its main pharmacological target is the competitive inhibition of dihydrofolatereductase (DHFR), an intracellular enzyme that reduces folic acids to key intermediates in several important biochemical pathways of DNA and RNA synthesis (Gonen and Assaraf 2012). Unfortunaltely, the resistance in cancer cells often compromises the efficacy of the anticancer therapy with MTX (Zhao and Goldman 2003). In addition, the conventional MTX delivery strategies often lead to undesirable shortcomings, such as the severe side effects associate with the treatment, including neurologic toxicity, renal failure and mucositis (Banerjee et al. 2002; Rubino 2001).

Chitosan (CS) is a naturally occurring polymer that has been attracting increasing attention in pharmaceutical and biomedical applications because of its biocompatibility, biodegradability, non-toxicity, cationic properties and bioadhesive characteristics (Bao et al. 2008; Fan et al. 2012). Therefore, CS nanoparticles (NPs) are of great interest as drug delivery systems, including applications for cancer therapy (Chen et al. 2014; Deng et al. 2014; Trapani et al. 2011). The ionotropic gelation technique is particularly suitable for the incorporation of pharmaceuticals, as it can be achieved in aqueous conditions (Agnihotri et al. 2004; Berger et al. 2004; Fan et al. 2012). Moreover, the formation process is solely based on the electrostatic interaction of oppositely charged compounds and, thus, it is not necessary any chemical modification. CS-based NPs containing MTX were already reported in the literature, but most of them with drawback of using a cross-linked agent (Chen et al. 2014; Jia et al. 2014; Luo et al. 2014; Wu et al. 2009). Likewise, there is considerable progress already achieved regarding the mechanisms underlying drug release from the nanostructures, being most of the current release methods based on reactions that commonly occur in response to environmental factors, 
i.e. the acidic conditions of subcellular compartments ( $\mathrm{Li}$ et al. 2012). Therefore, the knowledge about this mechanism gave basis for the research of $\mathrm{pH}$-responsive nanocarriers.

Upon this sense, and in an attempt to develop an efficient drug delivery device for cancer therapy, here we reported a simple strategy for preparation of $\mathrm{pH}$-responsive MTX-loaded CSbased NPs by using the ionic gelation method. Without crosslinkers and/or organic solvents, a bioactive amino acid-based surfactant with $\mathrm{pH}$-sensitive properties was incorporated in the complexation process during NP preparation. This compound was added as a modifier into the formulation in order to achieve a greater antitumor activity of the encapsulated drug. The anionic amphiphile derived from $\mathrm{N}^{\alpha}, \mathrm{N}^{\varepsilon}$-dioctanoyl lysine with a sodium counterion (77KS) have pH-sensitive membrane-lytic behavior and low cytotoxicity (Nogueira et al. 2011a), suggesting that it may be a specific adjuvant with ability to destabilize the endosomal membrane in the mildly acidic environment and, thus, release more specifically and efficiently the drug inside the malignant cells (Lee et al. 2008). Our group have previously published a study on the design of NPs based on medium molecular weight (MMW) CS and incorporating a lysine-based surfactant with a different counterion (77KL, with lithium counterion) (Nogueira et al., 2013). This study showed promising results, which therefore provided us the basis to continue the research in this field, seeking for important progress in delivery strategies.

Finally, besides the preparation and characterization of CS-based NPs incorporating the surfactant $77 \mathrm{KS}$, we evaluated the role of $\mathrm{pH}$ in the membrane-lytic activity of NPs and also in the in vitro drug release profile. In addition, to gain insight into the potential antitumor activity of NPs, the antiproliferative activity of both free and encapsulated drug was assessed using in vitro tumor cell models.

\section{Experimental}

Chemicals and reagents

Chitosan (CS) of low molecular weight (LMW) (deacetylation degree, 75-85\%; viscosity, 20-300 cP according to the manufacturer's data sheet), pentasodium tripolyphosphate (TPP), 2,5-diphenyl-3,-(4,5-dimethyl-2-thiazolyl) tetrazolium bromide (MTT), neutral red (NR) dye, dimethyl sulfoxide (DMSO), phosphate buffered saline (PBS) and trypsin-EDTA solution ( $0.5 \mathrm{~g}$ porcine trypsin and $0.2 \mathrm{~g}$ EDTA $-4 \mathrm{Na}$ per liter of Hanks' Balanced Salt Solution) were obtained from Sigma-Aldrich (St. Louis, MO, USA). Methotrexate (MTX, state purity 100.1 \%) was purchased from SM Empreendimentos Farmacêuticos Ltda. (São Paulo, SP, Brazil). 
Acetonitrile was purchased from Tedia (Fairfield, USA). Fetal bovine serum (FBS) and Dulbecco's Modified Eagle's Medium (DMEM), supplemented with L-glutamine (584 mg/l) and antibiotic/antimicotic (50 mg/ml gentamicin sulphate and $2 \mathrm{mg} / \mathrm{l}$ amphotericin B), were purchased from Vitrocell (Campinas, SP, Brazil). All other reagents were of analytical grade.

Surfactant included in the Nanoparticles

An anionic amino acid-based surfactant derived from $\mathrm{N}^{\alpha}, \mathrm{N}^{\varepsilon}$-dioctanoyl lysine and with an inorganic sodium counterion (77KS) was included in the NP formulation. It has a molecular weight of $421.5 \mathrm{~g} / \mathrm{mol}$, a critical micellar concentration (CMC) of $3 \times 10^{3} \mu \mathrm{g} / \mathrm{ml}$ and its chemical structure is formed by two alkyl chains, each one with eight carbon atoms (Sánchez et al. 2006, 2007). This surfactant was synthesized as previously described (Vives et al. 1999).

Nanoparticle preparation

CS-NPs were spontaneously obtained by ionotropic gelation technique, according to the methodology previously developed by Calvo et al. (1997) but with some modifications. The NPs were prepared with a selective CS:TPP:77KS ratio of 5:1:0.5 (w/w/w). Likewise, NPs omitting the surfactant and, thus, with CS:TPP ratio of 5:1 (w/w), were also prepared in order to perform comparative studies. Unloaded CS-NPs (unloaded-CS-NPs) were prepared by dropwise addition of a premixed solution containing the cross-linker TPP and the surfactant $77 \mathrm{KS}$ (both at $0.1 \%$, w/v) over the CS solution under magnetic stirring. Agitation was maintained for $20 \mathrm{~min}$, under room temperature and dark conditions, to allow the complete formation of NPs. The CS solution was prepared at a concentration of $0.1 \%(\mathrm{w} / \mathrm{v})$ and was dissolved in acetic acid solution (1\%, v/v). The $\mathrm{pH}$ of the $\mathrm{CS}$ final solution was adjusted to 5.5 with $1 \mathrm{M} \mathrm{NaOH}$ (Gan et al. 2005). Similarly, MTX-loaded CS-NPs (MTX-CS-NPs) were prepared following the same procedure, but adding MTX to a premixed TPP and 77KS solution, providing a final concentration of $0.07 \%(\mathrm{w} / \mathrm{v})$ of the antitumor drug (final ratio TPP:77KS:MTX 1:0.5:1, w/w/w).

Nanoparticle characterization 
The mean hydrodynamic diameter and the polydispersity index (PDI) of the NPs were determined by dynamic light scattering (DLS) using a Malvern Zetasizer ZS (Malvern Instruments, Malvern, UK), without any dilution of the samples. Each measurement was performed using at least three sets of ten runs. The zeta potential (ZP) values of the NPs were assessed by determining electrophoretic mobility with the Malvern Zetasizer ZS equipment. The measurements were performed after dilution of the formulations in $10 \mathrm{mM} \mathrm{NaCl}$ aqueous solution (1:10 volume per volume), using at least three sets of 10 runs. The $\mathrm{pH}$ measurements were determined directly in the NP suspensions, using a calibrated potentiometer (UB-10; Denver Instrument, Bohemia, NY, USA), at room temperature. Finally, the spectral properties of the model drug were assessed before its encapsulation and also after extraction from the NP structure. This assay was performed in order to verify the stability of MTX after entrapment into the NP matrix. The experiments were performed on a double-beam UV-VIS spectrophotometer (Shimadzu, Japan) model UV-1800, with a fixed slit width (2 nm) and a 10 mm quartz cell was used to obtain spectrum and absorbance measurements. The diluent optimized was $0.1 \mathrm{M}$ sodium hydroxide.

\section{Drug entrapment efficiency}

The drug association efficiency was determined by the ultrafiltration/centrifugation technique using Amicon Ultra-0.5 Centrifugal Filters (10000 Da MWCO, Millipore). The entrapment efficiency (EE\%) was calculated by the difference between the total concentration of MTX found in the NP suspension after its complete dissolution in methanol, and the free MTX concentration determined in the ultrafiltrate after separation of the NPs. The EE\% of MTX in the NPs was measured using a previously validated reversed-phase high performance liquid chromatography (RP-HPLC) method (Nogueira et al. 2014a). The chromatographic analyses were carried out on a Shimadzu LC system (Shimadzu, Kyoto, Japan), using a Waters XBridgeTM C18 column ( $250 \mathrm{~mm}$ x $4.6 \mathrm{~mm}$ I.D., $5 \mu \mathrm{m}$ ), a mobile phase consisted of potassium phosphate buffer (0.05 M, pH 3.2): acetonitrile (86:14, v/v) and UV detection set at $303 \mathrm{~nm}$. The analytical method showed acceptable results for the specificity test (no interference of the NP components on the quantitative analysis), and also provided good linearity in the $1-30$ $\mu \mathrm{g} / \mathrm{ml}$ concentration range $(\mathrm{r}=0.9999)$, precision (with a relative standard deviation lower than $1.5 \%$ ) and accuracy (mean recovery of $99.39 \%$ ).

In vitro release study 
In vitro release assessments from MTX-loaded CS-NPs were carried out for $480 \mathrm{~min}$ in PBS at pH 7.4, 6.6 and 5.4. An aliquot of NPs $(1 \mathrm{ml})$ was placed in a dialysis bag (SigmaAldrich, $14000 \mathrm{MWCO}$ ) and suspended in $50 \mathrm{ml}$ of PBS at $37^{\circ} \mathrm{C}$ under gentle magnetic stirring (100 rpm). The withdrawal of $2 \mathrm{ml}$ of the external medium from the system was done at predetermined time interval and was filtered through a $0.45-\mu \mathrm{m}$ membrane. An equal volume of fresh medium was replaced in order to maintain the sink conditions. The release of the free drug was also investigated in the same way. The cumulative release percentage of MTX at each time point was estimated by the RP-HPLC method described previously (Nogueira et al. 2014a), using analytical curves obtained with the release medium (PBS at pH 7.4, 6.6 and 5.4) as diluents.

pH-dependent membrane-lytic activity of nanoparticles

The pH-dependent membrane-lytic activity of NPs was assessed using the hemolysis assay, with the erythrocytes as a model of the endosomal membrane (Akagi et al. 2010; Wang et al. 2007). Erythrocytes were isolated from human blood, which was obtained from discarding samples of the Clinical Analysis Laboratory of the University Hospital of Santa Maria. Tubes containing EDTA as anticoagulant were used for blood collection. Erythrocytes ( $25 \mu 1$ of the suspension prepared in PBS) were incubated with increasing concentrations of unloaded-CSNPs and MTX-CS-NPs suspended in PBS buffer of $\mathrm{pH} 7.4,6.6$ or 5.4. The extent and kinetics of hemolysis were assessed as reported earlier (Nogueira et al. 2011a). Absorbance of the hemoglobin released in supernatants was measured at $540 \mathrm{~nm}$ by a Shimadzu UV-1800 spectrophotometer (Shimadzu, Kyoto, Japan).

Cytotoxicity assays - antitumor activity of free and encapsulated drug

The tumor cell lines HeLa (human epithelial cervical cancer) and MCF-7 (human breast cancer) were used as in vitro models to study the antitumor activity of MTX in its free and nanoencapsulated forms. Both cells were grown in DMEM medium (4.5 g/l glucose), supplemented by $10 \%(\mathrm{v} / \mathrm{v}) \mathrm{FBS}$, at $37^{\circ} \mathrm{C}$ with $5 \% \mathrm{CO}_{2}$. These cells were routinely cultured in $75 \mathrm{~cm}^{2}$ culture flasks and were harvested using trypsin-EDTA when the cells reached approximately $80 \%$ confluence. 
HeLa $\left(7.5 \times 10^{4}\right.$ cells $\left./ \mathrm{ml}\right)$ and MCF-7 $\left(1 \times 10^{5}\right.$ cells $\left./ \mathrm{ml}\right)$ cells were seeded into the 60 central wells of 96-well cell culture plates in $100 \mu 1$ of complete culture medium. Cells were incubated for $24 \mathrm{~h}$ under $5 \% \mathrm{CO}_{2}$ at $37^{\circ} \mathrm{C}$ and the medium was then replaced with $100 \mu \mathrm{l}$ of fresh medium supplemented by 5\% (v/v) FBS containing the treatments. Unloaded-CS-NPs were assayed in the $25-300 \mu \mathrm{g} / \mathrm{ml}$ concentration range, being these concentrations based on the total composition of the formulation. In contrast, MTX-CS-NPs and free MTX were assessed in the $1-50 \mu \mathrm{g} / \mathrm{ml}$ concentration range and, in this case, the concentrations were based on the total amount of MTX in each sample. Untreated control cells were exposed to medium with 5\% (v/v) FBS only. The cell lines were exposed for $24 \mathrm{~h}$ to each treatment, and their viability was assessed by two different assays, MTT and NRU (NR uptake).

The MTT endpoint is a measurement of cell metabolic activity and the assay is based on the first protocol described by Mossmann (1983). On the other hand, the NRU assay is based on the protocol described by Borenfreund and Puerner (1985), and reflects the functionality of the lysosomes and cell membranes. After complete the treatment time ( $24 \mathrm{~h})$, the medium was removed, and $100 \mu \mathrm{l}$ of MTT in PBS $(5 \mathrm{mg} / \mathrm{ml})$ diluted 1:10 in medium without FBS was then added to the cells. Similarly, $100 \mu \mathrm{l}$ of $50 \mu \mathrm{g} / \mathrm{ml}$ NR solution in DMEM without FBS was added to each well for the NRU assay. The microplates were further incubated for $3 \mathrm{~h}$ under $5 \% \mathrm{CO}_{2}$ at $37^{\circ} \mathrm{C}$, after which the medium was removed, and the wells of the NRU assay were washed once with PBS. Thereafter, $100 \mu \mathrm{l}$ of DMSO was added to each well to dissolve the purple formazan product (MTT assay) or, similarly, $100 \mu \mathrm{l}$ of a solution containing $50 \%$ ethanol absolute and $1 \%$ acetic acid in distilled water was added to extract the NR dye (NRU assay). After 10 min shaking at room temperature, the absorbance of the resulting solution was measured at $550 \mathrm{~nm}$ using a SpectraMax M2 (Molecular Devices, Sunnyvale, CA, USA) microplate reader. Cell viability for MTT and NRU was calculated as the percentage of tetrazolium salt reduced by viable cells in each sample or as the percentage of uptake of NR dye by lysosomes, respectively. The viability values were normalized by the untreated cell control (cells with medium only).

Hemocompatibility studies

Erythrocytes were isolated from human blood, as described for the experiments of $\mathrm{pH}$ dependent membrane-lytic activity. Twenty-five microliter aliquots of erythrocyte suspension were exposed to unloaded-CS-NPs at concentrations of 100, 250 and $500 \mu \mathrm{g} / \mathrm{ml}$ (concentrations based on the total composition of the formulation), and to MTX-CS-NPs or free MTX at 
concentrations of 10, 25 and $50 \mu \mathrm{g} / \mathrm{ml}$ (concentrations based on the total amount of MTX). The samples were incubated at room temperature for 10 minutes or $1 \mathrm{~h}$ and then centrifuged at 10000 rpm for 5 min to stop the reaction. Absorbance of the hemoglobin released in supernatants was measured at $540 \mathrm{~nm}$ by a double-beam UV-VIS spectrophotometer (Shimadzu, Kyoto, Japan), model UV-1800.

Statistical analysis

Results are expressed as mean \pm standard error of the mean (SE) and statistical analyses were performed using one-way analysis of variance (ANOVA) to determine the differences between the datasets, followed by Tukey's post-hoc test for multiple comparisons, using SPSS ${ }^{\circledR}$ software (SPSS Inc., Chicago, IL, USA). All in vitro experiments were performed at least three times, using three replicate samples for each formulation concentration tested. $p<0.05$ was considered significant.

\section{Results and discussion}

The ionotropic gelation between CS, as the polycation, and TPP, as the polyanionic partner, leads to the formation of stable colloidal systems (Trapani et al. 2011). Therefore, using the optimized operating parameters, this simple and solvent-free procedure was chosen to prepare the NPs in this work. The 5:1 chitosan to TPP ratio was confirmed as a stable and suitable ratio to carry out the following studies, as also done by Calvo et al. (1997). Decreasing the chitosan to TPP ratio provokes an increasing turbidity, indicating a shift in the size variation of the particles to the larger end of the scale. Likewise, an excess of TPP may culminates in NP aggregation, once all the surface charges of CS have been annulled by excess polyanion (Fan et al. 2012).

Before NP preparation, the $\mathrm{pH}$ of CS solution was set at 5.5, as at this $\mathrm{pH}$ the NPs were smaller and have higher ZP (Gan et al. 2005), suggesting their greater stability. Additionally, about $90 \%$ of the amino groups of CS $(\mathrm{pKa}=6.5)$ are protonated at $\mathrm{pH} 5.5$ (Mao et al. 2010), which ensures that the crosslinking process takes place to form CS-NPs. Likewise, a pH value of 5.5 would ionize most of the carboxylic groups of MTX, which allows attractive electrostatic interactions between the negatively charged drug molecules and positively charged CS molecules. The MTX molecule is a polyelectrolyte carrying two carboxyl groups ( $\alpha$-carboxyl and $\gamma$-carboxyl with $\mathrm{pKa}$ of 3.4 and 4.7 , respectively), and a number of potentially protonated 
nitrogen functions, being the most basic with pKa of 5.7 (Rubino 2001). Finally, it is worth mentioning that due to its low water solubility, MTX was dissolved in the basic solution of TPP ( $\mathrm{pH} \sim 9.0)$ before crosslinking CS.

The choice of 77KS as a bioactive excipient in the NP formulation was based on our previous studies, which showed its $\mathrm{pH}$-sensitive membrane-disruptive activity, together with improved kinetics in the endosomal $\mathrm{pH}$ range and low cytotoxic potential (Nogueira et al. 2011a, 2011b). 77KS was incorporated into the NPs at a concentration below its CMC, thereby indicating that this surfactant is presented in the formulation in its monomer form. Moreover, our group has already demonstrated that the inclusion of another surfactant from the same family (77KL) in the composition of polymeric NPs improved their in vitro antitumoral activity and also gave them a pH-responsive behavior (Nogueira et al. 2013). It is noteworthy that this previous study gave us the basis to continuous the research in the field of nanocarriers with $\mathrm{pH}$ dependent properties.

\section{Characterization of Nanoparticles}

Firstly, the stability of the drug after its encapsulation was assessed through the spectral analysis, as shown in Fig. 1. The UV-vis spectrum of the drug extracted from NPs was similar to that obtained for MTX solution before encapsulation, which proved the integrity of MTX molecule after its entrapment into the NP matrix. In addition, the NPs were characterized for the mean particle size, PDI and ZP. Table 1 shows the characterization parameters of the NPs obtained in the presence and in the absence of MTX and/or 77KS. The hydrodynamic size of all NPs was in the range of 162 to $183 \mathrm{~nm}$, with no considerable variations due to the presence of the drug. The presence of $77 \mathrm{KS}$ is accompanied by an increase in the size of NPs, but without significant differences. It is worth pointing out that these NPs with LMW-CS and 77KS have a diameter almost 2-fold lower than the NPs prepared with MMW-CS and 77KL (Nogueira et al. 2013). The small size of nanocarriers may constitute a great feature to improve the drug delivery to the tumor tissues and, more specifically, to the intracellular compartments of tumor cells (Elbakry et al. 2012; Huang et al. 2012). Concerning the NP surface charge, positive ZP values (> $22 \mathrm{mV}$ ) were detected for all formulations. ZP is a measurement of the electric charge at the surface of the particles and indicates the physical stability of colloidal systems. It should be noted that there was a slight decrease in the surface charge due to the presence of the drug. This reduced ZP value can be attributed to the electrostatic complexation of MTX with the free amino groups of CS, which, therefore, diminishes the net positive charge in the NP surface. 
Likewise, the entrapment efficiency of MTX showed a clear dependence on the presence of the surfactant in the formulation. In the absence of $77 \mathrm{KS}$, the EE\% obtained ( 68\%) was significantly higher than the value obtained in the presence of 77KS $(\sim 38 \%)$. The high incorporation capacity of CS-NPS without 77KS (CS:TPP 5:1, w/w) is probably related to the chemical nature of the drug and to its interaction with the NP structure at the $\mathrm{pH}$ value of the experimental conditions. On the other hand, the lower encapsulation efficiency of the CS-NPs containing the surfactant (CS:TPP:77KS 5:1:0.5, w/w/w) might be attributed to the competition between MTX and 77KS, both negatively charged, for the electrostatic complexation with the positively charged CS molecules.

In vitro release study

The release profiles of MTX from the CS-NPs showed a first phase with an initial burst release of 57\% in the first $60 \mathrm{~min}$ at $\mathrm{pH} 7.4$, as shown in Fig. 2. This is possibly due to the drug molecules dispersing close to the NP surface. Considering the electrostatic attraction existing between the negatively charged drug and the positively charged polymer, we can assume that the MTX molecules are both adsorbed at the particle surface (Gan and Wang 2007) and entrapped/embedded into CS nano-matrix by hydrogen bonding and hydrophobic forces (Calvo et al. 1997). Therefore, the way by which the drug is associated with the carrier (adsorption or encapsulation) might determine its release rate from the matrix. In the second phase, a constant release of the drug was observed up to $480 \mathrm{~min}$, which can be a result of the diffusion of MTX through the polymer wall. A control experiment using free MTX was also carried out under similar conditions and complete diffusion across the dialysis membrane was found to occur within $240 \mathrm{~min}$.

When the studies were performed at acidic environment, the release rate was accelerated, which confirms the $\mathrm{pH}$-dependent release pattern of this nanostructure. The triggered release of payload under acidic conditions is probably attributed to the $\mathrm{pH}-$ responsive activity of $77 \mathrm{KS}$ (Nogueira et al. 2011a). The cumulative MTX release at pH 6.6 and 5.4 was in general significantly faster $(\mathrm{p}<0.05)$ than at $\mathrm{pH}$ 7.4. At $480 \mathrm{~min}, 96$ e $100 \%$ of MTX was released at pH 6.6 and 5.4, respectively, while only $89 \%$ of drug release was reached at $\mathrm{pH}$ 7.4. The total drug release at acidic conditions might be attributed to swelling and degradation of the compact NPs (Gan and Wang 2007). Additionally, noteworthy that the accelerated release at acidic conditions might promote an improved drug delivery in the acidic extracellular $\mathrm{pH}$ of tumors $\left(\mathrm{pH}_{\mathrm{e}} \sim 6.6\right)(\mathrm{Na}$ et al. 2003). Nevertheless, the $\mathrm{pH}$-dependent pattern observed here was not as 
effective as was in our previous work (Nogueira et al. 2013), in which it was used MMW-CS and the surfactant 77KL as bioactive adjuvant in the NP formulation. The faster release achieved here could be attributed to the formulation of NPs with LMW-CS, as it was previously described that the total drug release decreased significantly with increasing CS molecular weight (Gan and Wang 2007). Furthermore, these overall results could be attributed to the lower encapsulation efficiency obtained herein with the surfactant $77 \mathrm{KS}$.

pH-dependent membrane-lytic activity of nanoparticles

The hemolysis assay, with the erythrocyte as a model for the endosomal membrane, was applied herein to assess whether the presence of $77 \mathrm{KS}$ provides to the NPs a $\mathrm{pH}$-responsive membrane-lytic behavior. Firstly, it is worth mentioning that strong correlations have been reported between hemolytic activity and endosomal disturbance induced by membranedisruptive agents (Chen et al. 2009; Lee et al. 2010; Plank et al. 1994). This biological correlation justifies, thus, the use of erythrocytes as a model for endosomal membrane.

The membrane-lytic activity of the NPs as a function of concentration, with varying $\mathrm{pH}$ and incubation time, is shown in Fig. 3. Primarly, we evaluated the membrane lysis induced by unloaded and MTX-loaded CS-NPs incorporating 77KS (Fig. 3a, b, respectively). At physiological $\mathrm{pH}$, negligible hemolysis was observed after 10 min incubation, while 8.5 and $25 \%$ of hemolysis was observed after 60 min incubation with NPs loading or not the drug, respectively. As the $\mathrm{pH}$ decreased to 6.6 or 5.4 , the membrane-lytic activity of unloaded and MTX-loaded CS-NPs increased significantly $(\mathrm{p}<0.05)$ in a dose-dependent manner. After 60 min incubation, unloaded-CS-NPs were 2.81- and 3.17-fold more hemolytic at pH 6.6 and 5.4 than at pH 7.4, respectively. Likewise, and even more efficiently, MTX-CS-NPs were 4.06- and 9.35-fold more membrane-lytic active in environments simulating early and late endosomal compartments, respectively. Noteworthy that these results demonstrated that entrapment of MTX into NPs did not change their pH-responsive behavior. Finally, in order to confirm that the $\mathrm{pH}$-sensitive membrane-lytic activity of NPs was really attributed to the surfactant, unloaded and MTX-loaded CS-NPs were prepared without 77KS and submitted to the same assay procedure. Negligible or low hemolysis rates were obtained in all tested conditions, proving the lack of pH-responsive behavior of these NPs in the absence of 77KS (Fig. 3c, d, respectively). The hemolysis of non-encapsulated MTX was also assessed, but insignificant membrane lysis was obtained in the entire $\mathrm{pH}$ range (data not shown). 
Therapeutics agents, including antitumor drugs, act at intracellular sites and, thus, their clinical efficacy depends on efficient intracellular trafficking (Hu et al. 2007; Plank et al. 1998). As cells usually take up drug nanocarriers via endocytosis, an important challenge for the intracellular delivery of therapeutic compounds is to circumvent the non-productive trafficking from endosomes to lysosomes, where degradation may occur (Stayton et al. 2000). Therefore, the design of $\mathrm{pH}$-responsive nanostructures is considered a great approach to facilitate the drug release into the cytosol by destabilizing endosomal membranes under mildly acidic conditions (Chen et al. 2009). Agents commonly used to promote NP escape/release from endosomes include $\mathrm{pH}$-sensitive peptides and surfactants, $\mathrm{pH}$-buffering polymers, and fusogenic lipids (Li et al. 2012; Nogueira et al. 2014b).

Upon this sense, we prepare polymeric NPs incorporating a surfactant that showed high efficiency at disrupting cell membranes within the $\mathrm{pH}$ range characteristic of the endosomal compartments (Nogueira et al. 2011a). As expected, the NPs containing 77KS presented a clear membrane-lytic behavior dependent on $\mathrm{pH}$, suggesting their potential ability to promote triggered release of entrapped drug in a $\mathrm{pH}$-responsive fashion. The enhanced hemolysis induced by NPs at acidic conditions could be explained firstly, by the electrostatic attraction of the NPs to the negatively charged cell membranes and, secondly, by a modification in the hydrophobic/hydrophilic balance of $77 \mathrm{KS}$ at the $\mathrm{pH}$ range of endosomes. The carboxylic group of $77 \mathrm{KS}$ seems to become protonated at acidic conditions, which makes it non-ionic and enhances its hydrophobicity. These hydrophobic segments can insert into lipophilic regions of lipid bilayers, causing membrane solubilization or altering the permeability of the membrane, hence, inducing cell lysis.

In vitro assessments of antitumor activity

The in vitro antitumor activity of MTX-CS-NPs and free MTX were performed using two different tumor cell lines, HeLa and MCF-7. We chose the in vitro model systems to perform this study, as they provide a rapid and effective means to assess NPs for a number of toxicological endpoints and mechanism-driven responses. Primarly, we evaluated if the unloaded-CS-NPs have any cytotoxic effect, which can be attributed especially to the presence of surfactant (Nogueira et al. 2011b). Fig. 4a demonstrated that unloaded-CS-NPs have low or negligible cytotoxicity against both cell lines and displayed at least $80 \%$ of cell viability.

As a second step, we studied the antiproliferative activity of the NPs containing the drug, and our results clearly proved that the cytotoxicity of MTX-CS-NPs incorporating 77KS was 
greater than that of free drug against both tumor cells (Fig. 4b-e). By increasing drug concentration, antitumor activity of NPs became higher in comparison to the same concentration range of free MTX. Low to moderate cytotoxic effects were achieved for free MTX, whereas MTX encapsulated into NPs significantly reduced the cell growth. For example, it can be evaluated from Fig. 4d that, by MTT assay, the MCF-7 cell viability at the $50 \mu \mathrm{g} / \mathrm{ml}$ drug concentration was decreased from $71.14 \%$ for free MTX to $40.80 \%$ for MTX-CS-NPs $(30.34 \%$ decrease in cell viability, $\mathrm{p}<0.05$ ). Likewise, but with low intensity, the viability of HeLa cells was decreased from $79.87 \%$ for free MTX to $61.57 \%$ for MTX-CS-NPs (18.30\% decrease in cell viability, $\mathrm{p}<0.05$ ) (Fig. 4b). On the other hand, the NRU assay displayed lower sensitivity to detect the cytotoxic effects of free and encapsulated drug in both cell lines. No significant differences were observed in HeLa cells (Fig. 4c), but this assay also detected greater activity of NPs in MCF-7 cells at the highest concentrations assayed (Fig. 4e). Noteworthy that MTT was the most sensitive viability assay for detecting the cytotoxic effects of the NPs, regardless of the cell line. The MTT assay determines cell metabolic activity within the mitochondrial compartment, while NRU endpoint measures membrane integrity. This implies that NPs primarily exerted toxicity on the mitochondrial compartment after cellular internalization, while the cell membranes were affect only at a later stage. This finding could be related to the mechanism of action of the drug. MTX is a potent inhibitor of the enzyme DHFR, which plays a key role in the synthesis of purines and thymine and, thus, leads to a blockage of DNA and RNA synthesis, and eventually to cell death (Rubino 2001). Therefore, MTX is a chemotherapeutic agent that mainly inhibits cell proliferation by acting in the intracellular sites, which justifies its lower effects on cell membrane integrity.

Due to its high polarity (at the neutral $\mathrm{pH}$ of biological fluids it is present mainly in the doubly anionic species), MTX can cross the cell membrane only with difficulty and, therefore, is transported into the cells by a specific, high-affinity carrier system, or by a passive diffusion mechanism, which can be limited by the high ionization state of MTX at physiological pH (Rubino 2001). This mechanism could justify the general low activity of free MTX in comparison with its encapsulated form. Likewise, MTX does not exert its cytotoxic activity only towards neoplasic cells, but also targets other tissues, thus determining a range of side effects. Therefore, in order to overcome the undesirable toxicity of MTX and to circumvent the risks associated with the administration of the free drug, considerable research efforts have been directed toward novel nanotechnology-based MTX delivery approaches (Chen et al. 2007; Kuznetsova et al. 2012; Thomas et al. 2012). Here, the enhanced cytotoxicity of MTX via the $\mathrm{pH}$-responsive NPs means that a significant reverse effect of drug resistance could has occurred. 
Moreover, nanoparticulated systems may reduce the resistance effects that characterize many anticancer drugs by a mechanism of cell internalization of the drug by endocytosis, by lowering drug efflux from the cells and, in the case of $\mathrm{pH}$-responsive NPs, by allowing an effective drug release from the endosomes to cell cytosol (Dong and Mumper 2010). Therefore, the $\mathrm{pH}$ dependent membrane-lytic activity of the MTX-CS-NPs (see the previous section) seems to be directly related with their enhanced therapeutic efficacy compared to the native drug.

Finally, we can highlight that during this study we observed differences in the sensitivity of cell lines and viability assays to each treatment. In view of that, we support the idea that complementary assays based on various cell mechanisms, as well as a combination of cell culture models, are need to raise the information output and the reliability of the results.

Hemocompatibility studies

The assessment of the hemocompatibility of a nanocarrier is indispensable for frequent intravenous dosing (Kuznetsova and Vodovozova 2014). Therefore, as the proposed nanocarriers were intended for intravenous administration, we evaluated if they cause any toxicological reaction to the erythrocytes. The hemocompatibility of unloaded and MTX-loaded CS-NPs, both containing the surfactant 77KS, was studied by hemolysis experiments (Fig. 5). The release of hemoglobin was used to quantify their erythrocyte-damaging properties. The unloaded-CS-NPs were non-hemolytic (less than 5\%) at the lower concentrations, and showed slight hemolysis at the highest concentration and after $1 \mathrm{~h}$ incubation. In contrast, the NPs containing MTX were almost non-hemolytic regardless of the concentration and the incubation time (maximum 8\% hemolysis). Likewise, free MTX was non-hemolytic in all the conditions (data not shown). Fortunately, these results were in contrast with those previously reported for other nanoformulations containing MTX (Kuznetsova and Vodovozova 2014; Nogueira et al. 2013), in which the encapsulated drug displayed higher hemoreactivity than its free solution. Altogether, the obtained results suggest the hemocompatibility of the designed $\mathrm{pH}$-sensitive NPs.

\section{Conclusions}

In summary, we have demonstrated a simple and solvent-free approach to prepare $\mathrm{pH}$ responsive CS-NPs by incorporating a biocompatible amino acid-based surfactant in the ionotropic complexation process. The obtained NPs presented a suitable nanosize and displayed 
accelerated release of MTX by decreasing $\mathrm{pH}$ from 7.4 to 5.4. The inclusion of $77 \mathrm{KS}$ on the formulation clearly gives it a $\mathrm{pH}$-sensitive membrane-lytic behavior, which suggests these NPs as a potential intracellular delivery system. Outstandingly, $\mathrm{pH}$-responsive CS-NPs significantly improved the in vitro antitumor activity of MTX with respect to the free drug in MCF-7 cell culture and, to a lesser extent, in HeLa cells. Based on the overall results, the conclusion that may be reached from this study is that this nanocarrier system represents a promising strategy for the administration of antitumor drugs in cancer therapy, which merits further investigations under in vivo conditions to confirm this premise.

\section{Conflict of interest statement}

The authors state that they have no conflict of interest.

\section{Acknowledgments}

This research was supported by Projects 483264/2012-1 of the Conselho Nacional de Desenvolvimento Científico e Tecnológico (CNPq - Brazil) and MAT2012-38047-C02-01 of the Ministerio de Economía y Competitividad (Spain). Daniele R. Nogueira holds a Postdoctoral fellowship from PNPD-CAPES (Brazil). 


\section{References}

Agnihotri SA, Mallikarjuna NN, Aminabhavi TM (2004) Recent advances on chitosan-based micro- and nanoparticles in drug delivery. J Control Release 100:5-28. doi: 10.1016/j.jconrel.2004.08.010

Akagi T, Kim H, Akashi M (2010) pH-dependent disruption of erythrocyte membrane by amphiphilic poly(amino acid) nanoparticles. J Biomater Sci 21:315-328. doi: $10.1163 / 156856209 \times 418519$

Banerjee D, Mayer-Kuckuk P, Capiaux G, Budak-Alpdogan T, Gorlick R, Bertino JR (2002) Novel aspects of resistance to drugs targeted to dihydrofolate reductase and thymidylate synthase. Biochim Biophys Acta 1587:164-173. doi: 10.1016/S0925-4439(02)00079-0

Bao H, Li L, Zhang H (2008) Influence of cetyltrimethylammonium bromide on physicochemical properties and microstructures of chitosan-TPP nanoparticles in aqueous solutions. J Coll Interface Sci 328:270-277. doi: 10.1016/j.jcis.2008.09.003

Berger J, Reist M, Mayer JM, Felt O, Peppas NA, Gurny R (2004) Structure and interactions in covalently and ionically crosslinked chitosan hydrogels for biomedical applications. Eur J Pharm Biopharm 57:19-34. doi: 10.1016/S0939-6411(03)00161-9

Borenfreund E, Puerner JA (1985) Toxicity determined in vitro by morphological alterations and neutral red absorption. Toxicol Lett 24:119-124.

Calvo P, Remuñan-López C, Vila-Jato JL, Alonso MJ (1997) Chitosan and chitosan/ethylene oxide-propylene oxide block copolymer nanoparticles as novel carriers for proteins and vaccines. Pharm Res 14:1431-1436. doi: 10.1023/A:1012128907225

Chen YH, Tsai CY, Huang PY, Chang MY, Cheng PC, Chou CH, Chen DH, Wang CR, Shiau AL, Wu CL (2007) Methotrexate conjugated to gold nanoparticles inhibits tumor growth in a syngeneic lung tumor model. Mol Pharmac 4:713-722. doi: 10.1021/mp060132k

Chen R, Khormaee S, Eccleston ME, Slater NKH (2009) The role of hydrophobic amino acid grafts in the enhancement of membrane-disruptive activity of $\mathrm{pH}$-responsive pseudopeptides. Biomaterials 30:1954-1961. doi: 10.1016/j.biomaterials.2008.12.036

Chen J, Huang L, Lai H, Lu C, Fang M, Zhang Q, Luo X (2014) Methotrexate-loaded PEGylated chitosan nanoparticles: synthesis, characterization, and in vitro and in vivo antitumoral activity. Mol Pharm 11:2213-2223. doi: 10.1021/mp400269z

Das M, Sahoo SK (2011) Epithelial cell adhesion molecule targeted nutlin-3a loaded immunonanoparticles for cancer therapy. Acta Biomater 7:355-369. doi: 10.1016/j.actbio.2010.08.010 
Deng X, Cao M, Zhang J, Hu K, Yin Z, Zhou Z, Xiao X, Yang Y, Sheng W, Wu Y, Zeng Y (2014) Hyaluronic acid-chitosan nanoparticles for co-delivery of MiR-34a and doxorubicin in therapy against triple negative breast cancer. Biomaterials 35:4333-4344. doi: 10.1016/j.biomaterials.2014.02.006

Dong X, Mumper RJ (2010) Nanomedicinal strategies to treat multidrug-resistant tumors: current progress. Nanomedicine 5: 597-615. doi: 10.2217/nnm.10.35

Elbakry A, Wurster EC, Zaky A, Liebl R, Schindler E, Bauer-Kreisel P, Blunk T, Rachel R, Goepferich A, Breunig M (2012) Layer-by-layer coated gold nanoparticles: size-dependent delivery of DNA into cells. Small 8:3847-3856. doi: 10.1002/smll.201201112

ETPN (2015) The European Technology Platform on Nanomedicine. Available at http://www.etp-nanomedicine.eu/public.

Fan W, Yan W, Xu Z, Ni H (2012) Formation mechanism of monodisperse, low molecular weight chitosan nanoparticles by ionic gelation technique. Colloids Surf B Biointerfaces 90:21-27. doi: 10.1016/j.colsurfb.2011.09.042

Gan Q, Wang T, Cochrane C, McCarron P (2005) Modulation of surface charge, particle size and morphological properties of chitosan-TPP nanoparticles intended for gene delivery. Colloids Surf B Biointerfaces 44:65-73. doi:10.1016/j.colsurfb.2005.06.001

Gan Q, Wang T (2007) Chitosan nanoparticle as protein delivery carrier - Systematic examination of fabrication conditions for efficient loading and release. Colloids Surf B Biointerfaces 59:24-34. doi:10.1016/j.colsurfb.2007.04.009

Gonen N, Assaraf YG (2012) Antifolates in cancer therapy: structure, activity and mechanisms of drug resistance. Drug Resist Updat 15:183-210. doi: 10.1016/j.drup.2012.07.002

Hu Y, Litwin T, Nagaraja AR, Kwong B, Katz J, Watson N, Irvine DJ (2007) Cytosolic delivery of membrane-impermeable molecules in dendritic cells using $\mathrm{pH}$-responsive core-shell nanoparticles. Nano Lett 7:3056-3064. doi: 10.1021/n1071542i

Huang K, Ma H, Liu J, Huo S, Kumar A, Wei T, Zhang X, Jin S, Gan Y, Wang PC, He S, Zhang X, Liang X-J (2012) Size-dependent localization and penetration of ultrasmall gold nanoparticles in cancer cells, multicellular spheroids, and tumors in vivo. ACS Nano 6:4483-4493. doi: 10.1021/nn301282m

Jia M, Li Y, Yang X, Huang Y, Wu H, Huang Y, Lin J, Li Y, Hou Z, Zhang Q (2014) Development of both methotrexate and mitomycin C loaded PEGylated chitosan nanoparticles for targeted drug codelivery and synergistic anticancer effect. ACS Appl Mater Interfaces 6:11413-11423. doi: 10.1021/am501932s 
Kuznetsova NR, Sevrin C, Lespineux D, Bovin NV, Vodovozova EL, Meszaros T, Szebeni J, Grandfils C (2012) Hemocompatibility of liposomes loaded with lipophilic prodrugs of methotrexate and melphalan in the lipid bilayer. J Control Release 160:394-400. doi: 10.1016/j.jconrel.2011.12.010

Kuznetsova NR, Vodovozova EL (2014) Differential binding of plasma proteins by liposomes loaded with lipophilic prodrugs of methotrexate and melphalan in the bilayer. Biochemistry 79:797-804. doi: 10.1134/S0006297914080070

Lee ES, Gao Z, Bae YH (2008) Recent progress in tumor pH targeting nanotechnology. J Control Release 132:164-170. doi: 10.1016/j.jconrel.2008.05.003

Lee Y-J, Johnson G, Pellois J-P (2010) Modeling of the endosomolytic activity of HA2-TAT peptides with red blood cells and ghosts. Biochemistry 49:7854-7866. doi: 10.1021/bi1008408

Li Y, Wang J, Wientjes MG, Au JL (2012) Delivery of nanomedicines to extracellular and intracellular compartments of a solid tumor. Adv Drug Deliv Rev 6:29-39. doi: 10.1016/j.addr.2011.04.006

Luo F, Li Y, Jia M, Cui F, Wu H, Yu F, Lin J, Yang X, Hou Z, Zhang Q (2014) Validation of a Janus role of methotrexate-based PEGylated chitosan nanoparticles in vitro. Nanoscale Res Lett 9:363. doi: 10.1186/1556-276X-9-363

Mao S, Sun W, Kissel T (2010) Chitosan-based formulations for delivery of DNA and siRNA. Adv Drug Deliv Rev 2010;62:12-27. doi: 10.1016/j.addr.2009.08.004

Mosmann T (1983) Rapid colorimetric assay to cellular growth and survival: application to proliferation and cytotoxicity assays. J Immunol Methods 65:55-63. doi:10.1016/00221759(83)90303-4

Na K, Lee ES, Bae YH (2003) Adriamycin loaded pullulan acetate/sulfonamide conjugate nanoparticles responding to tumor $\mathrm{pH}$ : $\mathrm{pH}$-dependent cell interaction, internalization and cytotoxicity in vitro. J Control Release 87:3-13. doi:10.1016/S0168-3659(02)00345-0

Nogueira DR, Mitjans M, Infante MR, Vinardell MP (2011a) The role of counterions in the membrane-disruptive properties of $\mathrm{pH}$-sensitive lysine-based surfactants. Acta Biomater 7:2846-2856. doi: 10.1016/j.actbio.2011.03.017

Nogueira DR, Mitjans M, Infante MR, Vinardell MP (2011b) Comparative sensitivity of tumor and non-tumor cell lines as a reliable approach for in vitro cytotoxicity screening of lysinebased surfactants with potential pharmaceutical applications. Int J Pharm 420:51-58. doi: 10.1016/j.ijpharm.2011.08.020 
Nogueira DR, Tavano L, Mitjans M, Pérez L, Infante MR, Vinardell MP (2013) In vitro antitumor activity of methotrexate via $\mathrm{pH}$-sensitive chitosan nanoparticles. Biomaterials 34:2758-2772. doi: 10.1016/j.biomaterials.2013.01.005

Nogueira DR, Macedo LB, Scheeren LE, Mitjans M, Infante MR, Rolim CMB, Vinardell MP (2014a) Determination of Methotrexate in pH-Sensitive Chitosan Nanoparticles by Validated RP-LC and UV Spectrophotometric Methods. J Appl Biopharm Pharmacokinet. In press.

Nogueira DR, Mitjans M, Vinardell MP (2014b) Nanotechnology approaches to target rndosomal $\mathrm{pH}$ : a promising strategy for an efficient intracellular drug, gene and protein delivery. Drug Deliv Lett 4:2-11. doi: 10.2174/221030310401140410115042

Plank C, Oberhauser B, Mechtler K, Koch C, Wagner E (1994) The influence of endosomedisruptive peptides on gene transfer using synthetic virus-like gene transfer systems. J Biol Chem 269:12918-12924.

Plank C, Zauner W, Wagner E (1998) Application of membrane-active peptides for drug and gene delivery across cellular membranes. Adv Drug Deliv Rev 34:21-35. doi: 10.1016/S0169-409X(98)00005-2

Rubino FM (2001) Separation methods for methotrexate, its structural analogues and metabolites. J Chromatogr B 764:217-254. doi: 10.1016/S0378-4347(01)00402-9

Sánchez L, Mitjans M, Infante MR, Vinardell MP (2006) Potential irritation of lysine derivative surfactants by hemolysis and HaCaT cell viability. Toxicol Lett 161:53-60. doi: 10.1016/j.toxlet.2005.07.015

Sánchez L, Mitjans M, Infante MR, García MT, Manresa MA, Vinardell MP (2007) The biological properties of lysine-derived surfactants. Amino Acids 32:133-136. doi: 10.1007/s00726-006-0318-x

Stayton PS, Hoffman AS, Murthy N, Lackey C, Cheung C, Tan P, Klumb LA, Chilkoti A, Wilbur FS, Press OW (2000) Molecular engineering of proteins and polymers for targeting and intracellular delivery of therapeutics. J Control Release 65:203-220. doi: 10.1016/S0168-3659(99)00236-9

Thomas TP, Huang BH, Choi SK, Silpe JE, Kotlyar A, Desai AM, Zong H, Gam J, Joice M, Baker JR (2012) Polyvalent dendrimer-methotrexate as a folate receptor-targeted cancer therapeutic. Mol Pharmac 9:2669-2676. doi: 10.1021/mp3002232

Trapani A, Denora N, Iacobellis G, Sitterberg J, Bakowsky U, Kissel T (2011) Methotrexateloaded chitosan and glycolchitosan-based nanoparticles: a promising strategy for the 
administration of the anticancer drug to brain tumors. AAPS Pharm Sci Tech 12:13021311. doi: 10.1208/s12249-011-9695-X

Vives MA, Infante MR, Gracia E, Selve C, Maugras M, Vinardell MP (1999) Erythrocyte hemolysis and shape changes induce by new lysine-derivate surfactants. Chem Biol Interact 118:1-18. doi: 10.1016/S0009-2797(98)00111-2

Wang X-L, Ramusovis S, Nguyen T, Lu Z-R (2007) Novel polymerizable surfactants with pHsensitive amphiphilicity and cell membrane disruption for efficient siRNA delivery. Bioconj Chem 18:2169-2177. doi: 10.1021/bc700285q

Wu P, He X, Wang K, Tan W, He C, Zheng M (2009) A novel methotrexate delivery system based on chitosan-methotrexate covalently conjugated nanoparticles. J Biomed Nanotechnol 5:557-564. doi: http://dx.doi.org/10.1166/jbn.2009.1073

Zhao R, Goldman ID (2003) Resistance to antifolates. Oncogene 22:7431-7457. doi: 10.1038/sj.onc. 1206946 
Table 1. Characterization parameters of the different CS-based NPs obtained in the presence and in the absence of MTX and/or the surfactant 77KS

\begin{tabular}{lcccc}
\hline & $\begin{array}{c}\text { CS-NPs } \\
(\mathrm{CS}: \text { TPP:77KS })\end{array}$ & $\begin{array}{c}\text { MTX-CS-NPs } \\
(\mathrm{CS}: \text { TPP:77KS })\end{array}$ & $\begin{array}{c}\text { CS-NPs } \\
(\mathrm{CS}: \mathrm{TPP})\end{array}$ & $\begin{array}{c}\text { MTX-CS-NPs } \\
(\mathrm{CS}: \mathrm{TPP})\end{array}$ \\
\hline $\begin{array}{l}\text { Hydrodynamic size } \\
(\mathrm{nm}) \pm \mathrm{SD}^{*}\end{array}$ & $183.0 \pm 1.234$ & $182.7 \pm 3.449$ & $162.9 \pm 1.405$ & $174.4 \pm 3.027$ \\
$\mathrm{PDI} \pm \mathrm{SD} *$ & $0.206 \pm 0.015$ & $0.225 \pm 0.022$ & $0.221 \pm 0.02$ & $0.223 \pm 0.013$ \\
$\begin{array}{l}\text { Zeta potential }(\mathrm{mV}) \\
\pm \text { SD* }\end{array}$ & $30.7 \pm 1.21$ & $22 \pm 0.569$ & $29.4 \pm 2.08$ & $23.5 \pm 1.29$ \\
pH & 5.55 & 5.53 & 5.52 & 5.53 \\
Entrapment & - & $38.4 \%$ & - & $68.02 \%$ \\
Efficiency (EE\%) & & & & \\
* SD = standard deviation & & & &
\end{tabular}




\section{Figure captions:}

Fig. 1 UV-vis spectra of (1) MTX free solution and (2) MTX extracted from CS-NPs.

Fig. $2 \mathrm{pH}$-dependent in vitro cumulative release of MTX from NPs in PBS buffer at pH 7.4, 6.6 and 5.4. Results are expressed as the mean \pm SE of three independent experiments. Statistical analyses were performed using ANOVA followed by Tukey's multiple comparison test. ${ }^{\text {a }}$ Significantly different from PBS pH $7.4(\mathrm{p}<0.05)$ and ${ }^{\mathrm{b}}$ significantly different from PBS $\mathrm{pH}$ $6.6(\mathrm{p}<0.05)$.

Fig. 3 pH-responsive membrane-lytic activity of CS-NPs with and without 77KS: (a) unloadedCS-NPs with 77KS, (b) MTX-CS-NPs with 77KS, (c) unloaded-CS-NPs without 77KS and (d) MTX-CS-NPs without 77KS. NP-induced membrane-lysis was expressed as a function of $\mathrm{pH}$, concentration and incubation time. Results are expressed as the mean \pm SE of three independent experiments. Statistical analyses were performed using ANOVA followed by Tukey's multiple comparison test. ${ }^{a}$ Significantly different from $\mathrm{pH} 7.4(\mathrm{p}<0.05),{ }^{\mathrm{b}}$ significantly different from pH $7.4(\mathrm{p}<0.005),{ }^{\mathrm{c}}$ significantly different from $\mathrm{pH} 6.6(\mathrm{p}<0.05)$ and ${ }^{\mathrm{d}}$ significantly different from $\mathrm{pH} 6.6(\mathrm{p}<0.005)$.

Fig. 4 The effect of varying concentrations of free drug and NPs incorporating 77KS on the survival rates of HeLa and MCF-7 cell lines by using two different viability assays: (a) unloaded-CS-NPs; (b) and (c) represent free MTX and MTX-CS-NPs in HeLa cells by MTT and NRU assays, respectively; (d) and (e) represent free MTX and MTX-CS-NPs in MCF-7 cells by MTT and NRU assays, respectively. Data are expressed as the mean of three independent experiments \pm SE. Statistical analyses were performed using ANOVA followed by Tukey's multiple comparison test. ${ }^{a}$ Significantly different from HeLa cells $(\mathrm{p}<0.05)$. $* \mathrm{p}<$ 0.05 and $* * \mathrm{p}<0.01$ denote significant differences from free MTX.

Fig. 5 Percentage of hemolysis caused by NPs incorporating 77KS after 10 and 60 min of incubation with human erythrocytes: (a) unloaded-CS-NPs and (b) MTX-CS-NPs. Each value represents the mean $\pm \mathrm{SE}$ of three experiments. 
Figure 1.

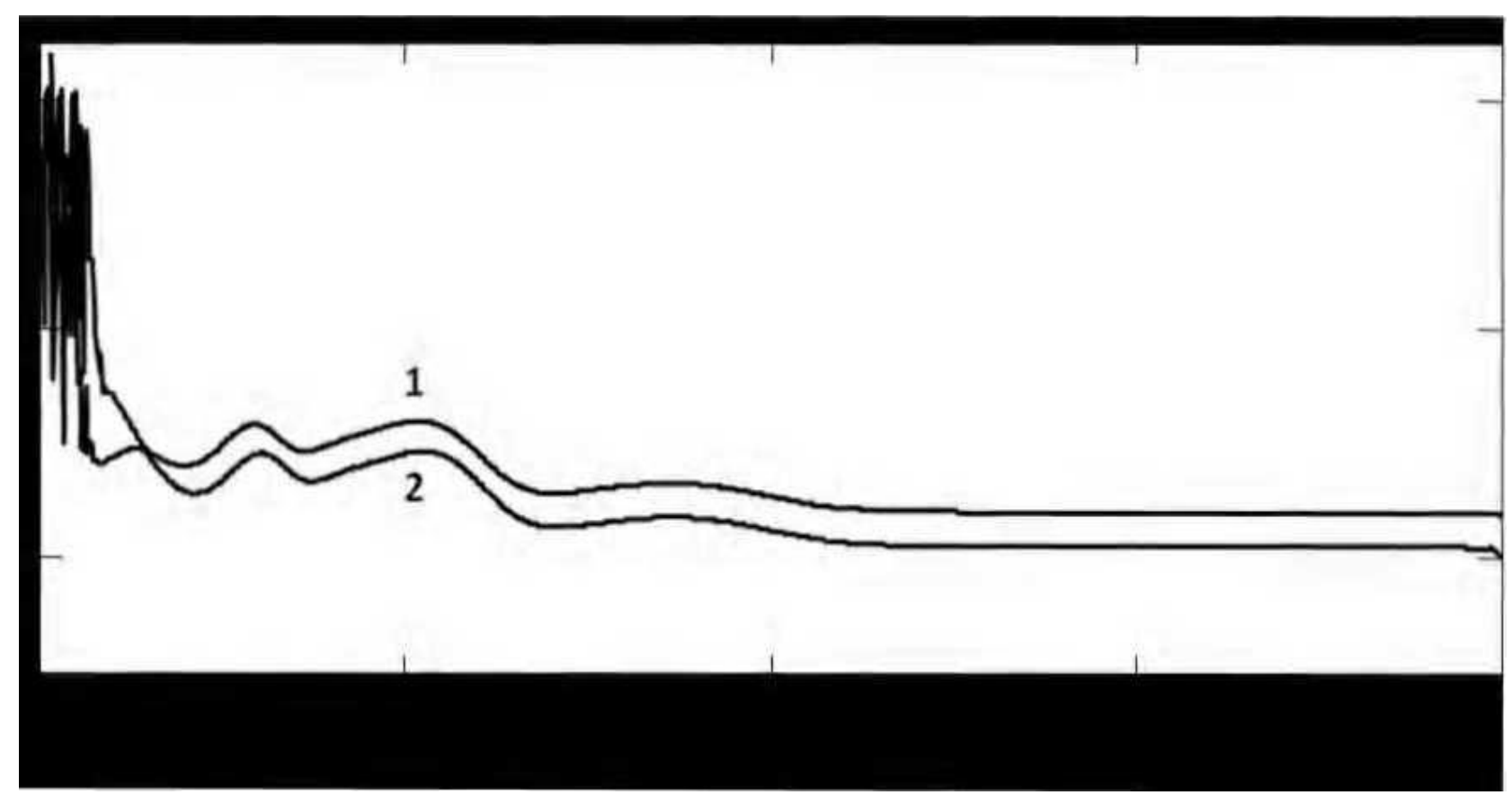


Figure 2

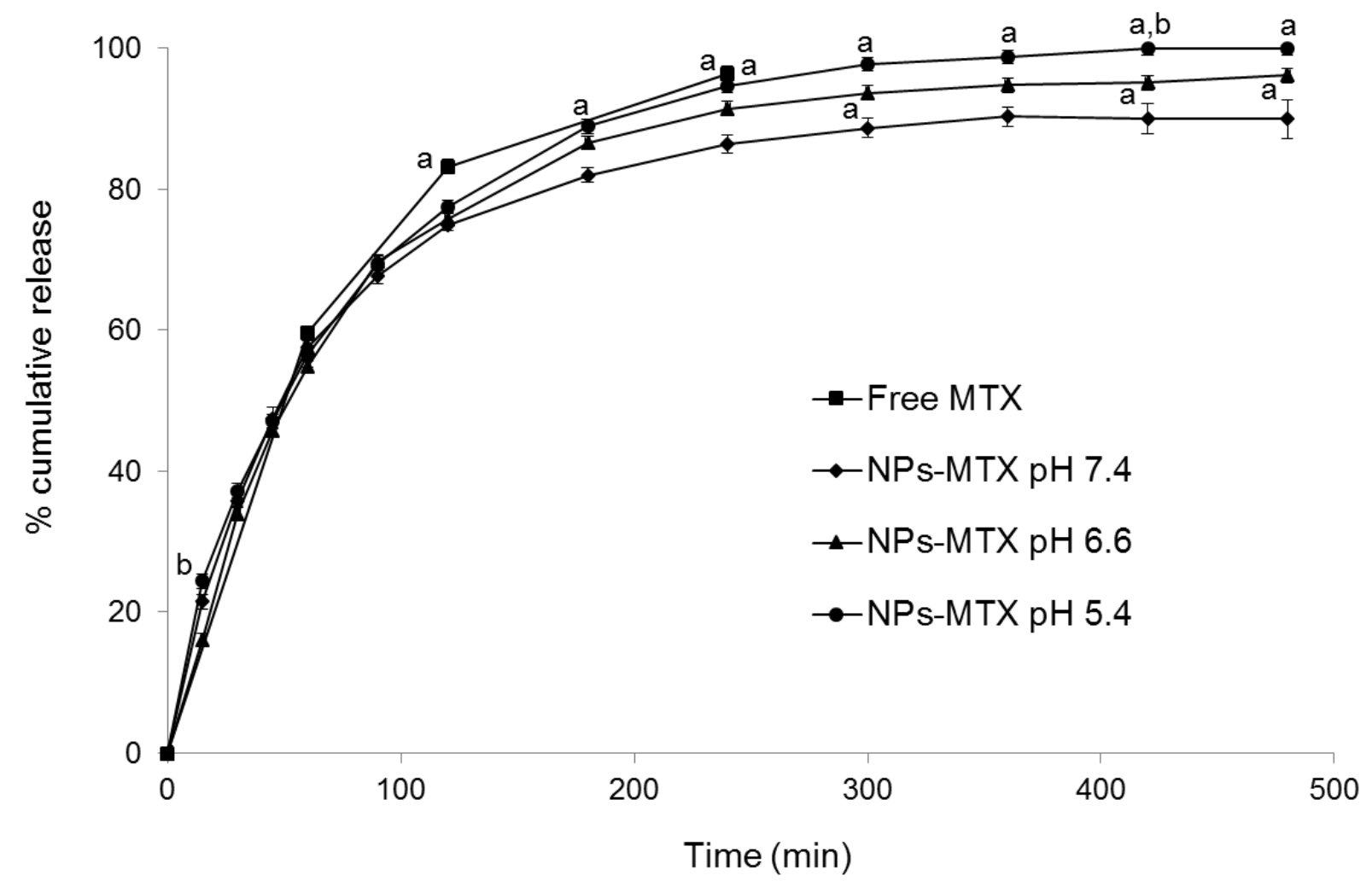


Figure 3.
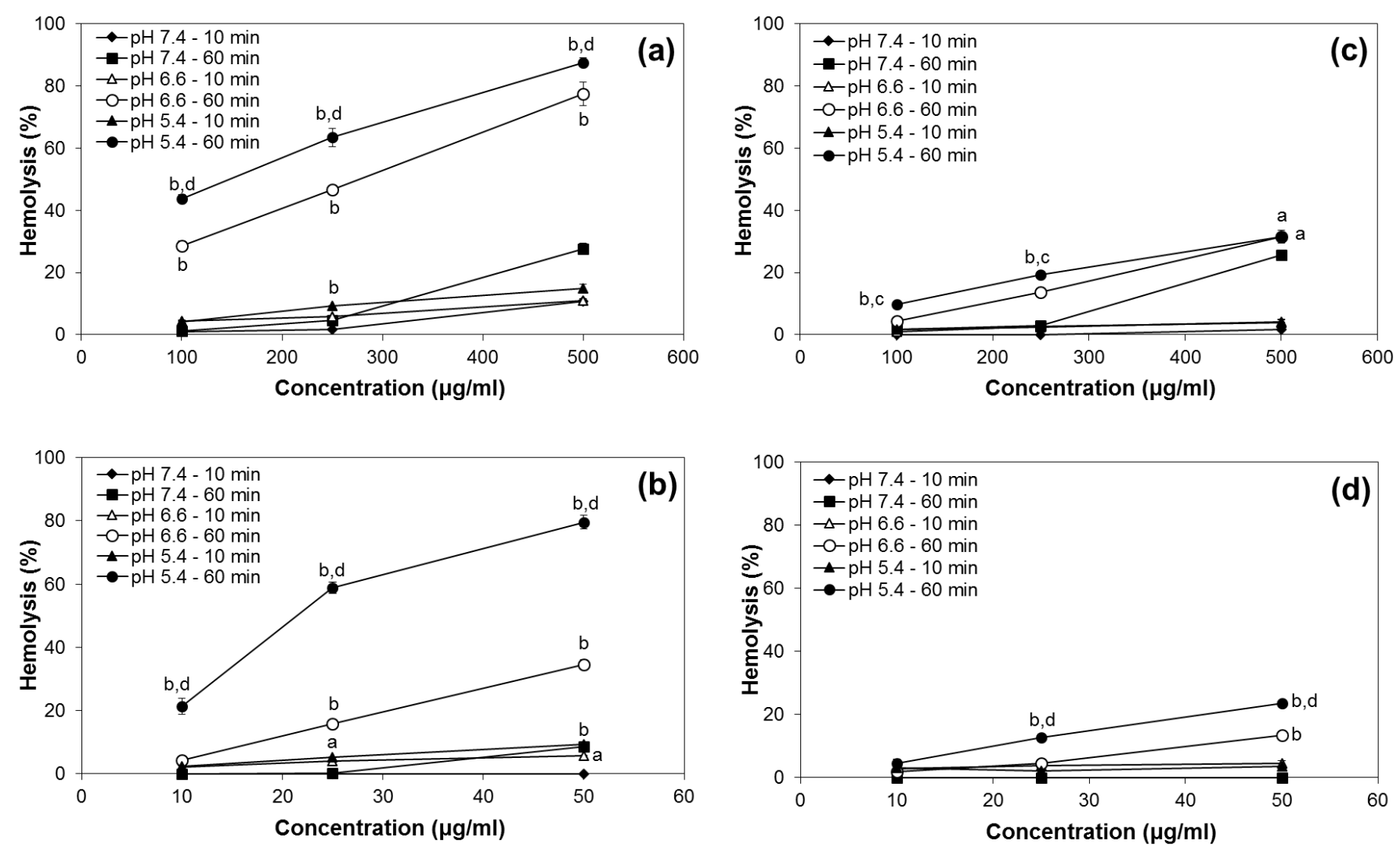
Figure 4.
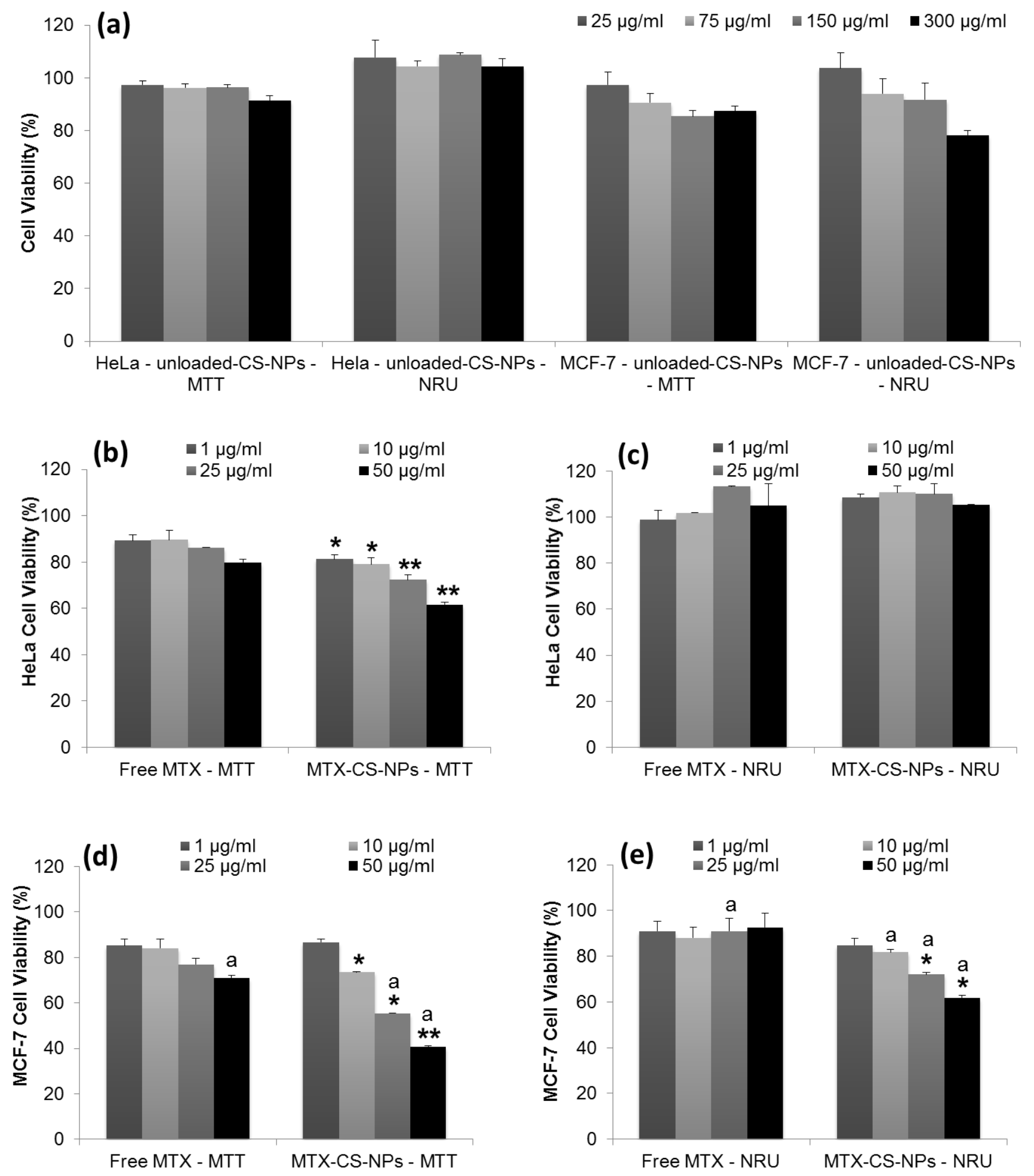
Figure 5.
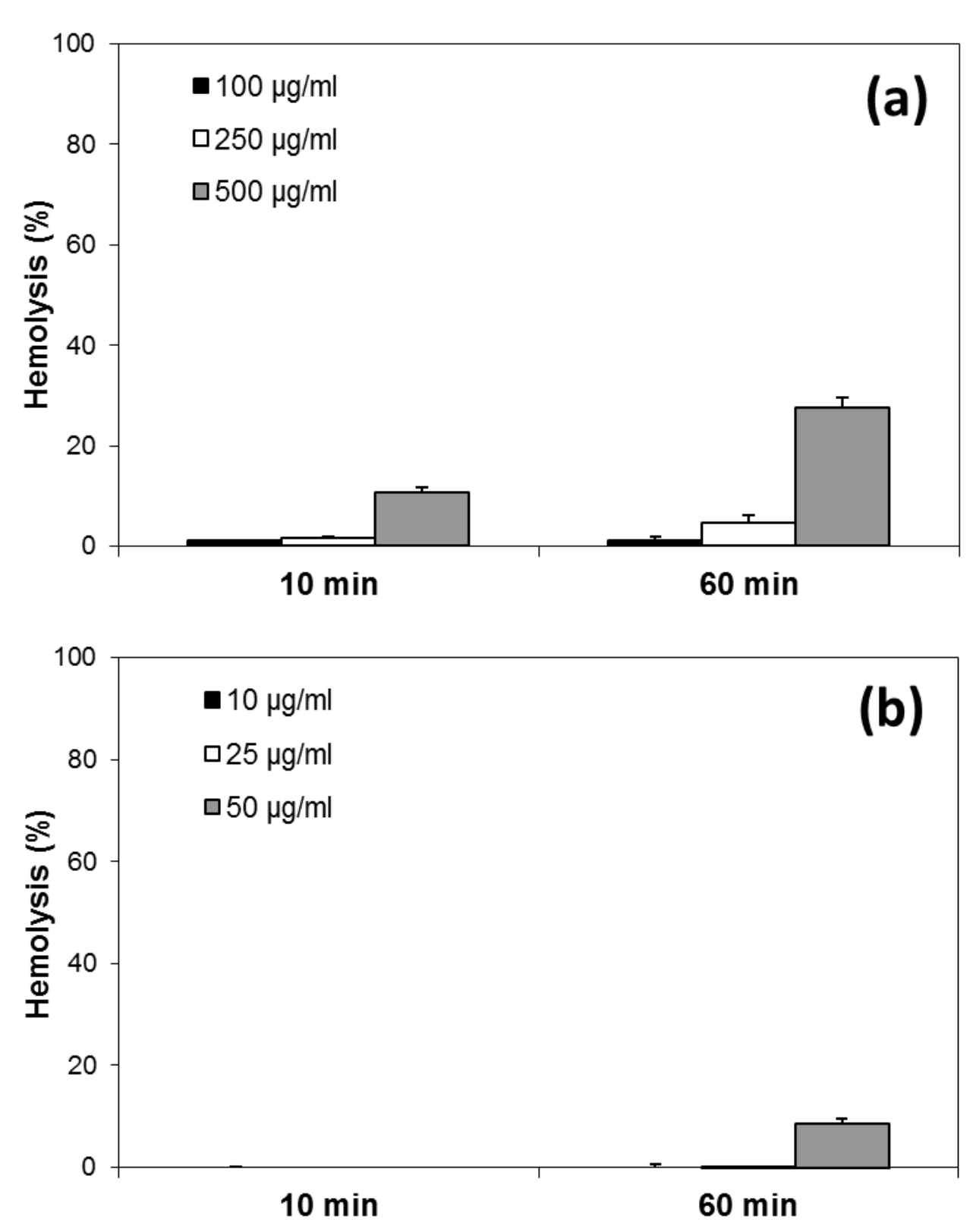